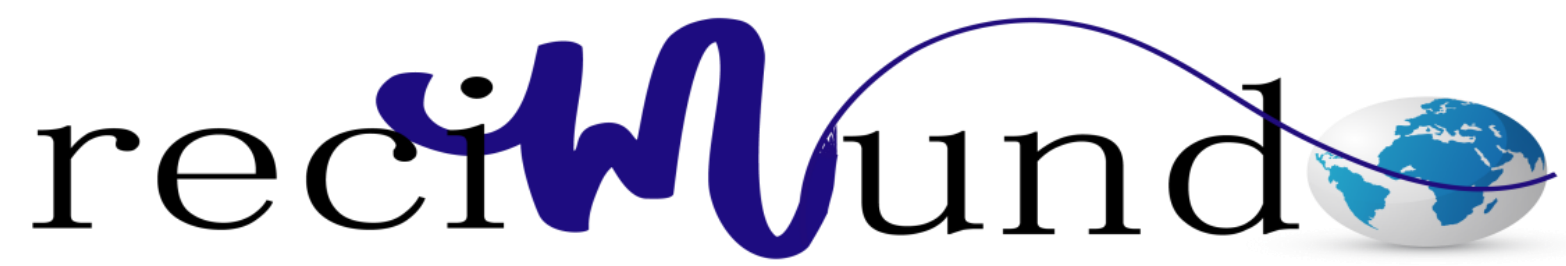

Revista Científica Mundo de la Investigación y el Conocimiento

Juan Patricio Méndez Castro a ; Xiomara Eunisse Mejía Escalante ${ }^{\text {b}}$; Olivia Mabell

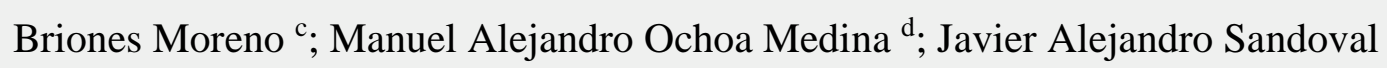
Lema ${ }^{e}$; Bryan Anthony Guerra Jaime ${ }^{\mathrm{f}}$

Tratamiento y cuidados de los infantes menores de 2 años con dermatitis atópica Treatment and care of infants under 2 years with atopic dermatitis

Revista Científica Mundo de la Investigación y el Conocimiento. Vol. 3 núm.3. Esp., noviembre, ISSN: 2588-073X, 2019, pp. 460-480

DOI: $10.26820 /$ recimundo/3.(3.Esp).noviembre.2019.460-480

URL: http://recimundo.com/index.php/es/article/view/616

Código UNESCO: 3205 Medicina Interna

Tipo de Investigación: Artículo de Revisión

(C) RECIMUNDO; Editorial Saberes del Conocimiento, 2019

Recibido: 15/09/2019

Aceptado: 23/10/2019

Publicado: 30/11/2019

Correspondencia: juanpmc01@gmail.com

a. Médico Cirujano; Hospital General IESS de Quevedo; juanpmc01@ gmail.com; Quevedo - Ecuador.

b. Médico; Hospital General IESS de Quevedo; xiomarita_0988@ hotmail.com; Quevedo - Ecuador.

c. Doctora en Medicina; Hospital General IESS de Quevedo; Quevedo - Ecuador

d. Médico General en Funciones Hospitalarias - Hospital Gustavo Domínguez; Santo Domingo, Ecuador; manuel_ochoa_18@hotmail.com

e. Médico; Médico General en Funciones Hospitalarias - Hospital Carlos Andrade Marín; alejojavier.89@gmail.com; Quito - Ecuador

f. Interno de Medicina - Hospital General Manta; bryanjaime@ @otmail.es; Manta - Ecuador 


\section{Tratamiento y cuidados de los infantes menores de 2 años con dermatitis \\ atópica}

Vol. 3, núm. 3 Esp., (2019)

Juan Patricio Méndez Castro; Xiomara Eunisse Mejía Escalante; Olivia Mabell Briones Moreno;

Manuel Alejandro Ochoa Medina; Javier Alejandro Sandoval Lema; Bryan Anthony Guerra

Jaime

\section{RESUMEN}

La dermatitis atópica es la enfermedad cutánea más frecuente en la infancia. Aproximadamente un $20 \%$ de los niños menores de dos años la padecen, incidencia que ha aumentado notablemente en los países desarrollados en los últimos años. Esta patología, es reconocida también, con el nombre de Eczema infantil; Dermatitis - atópica niños; Eczema - atópico - niños. Es una enfermedad inflamatoria de la piel que se manifiesta con lesiones rojizas que producen picor. Suelen aparecer en las zonas redondeadas en bebés (mentón, rodillas, codos, frente) y en el interior de los codos, detrás de las rodillas, pies, manos, torso y cuello en los niños. Representa una dolencia que afecta la calidad de vida del niño por lo que hay que llevarlo de manera inmediata al especialista, a fin de que el mismo indique de manera inmediata tratamiento dermatológico, tomando en consideración que, para ello, se deben tener en cuenta, por un lado, los cuidados generales y por otro, los tratamientos que incluyen fármacos de aplicación tópica o que se administran por vía oral. De manera general, los tratamientos indicados son corticoides tópicos, los cuales se utilizan para disminución de la inflamación de la piel, por lo que disminuyen el picor y el rascado. Existe una gran variedad de corticoides tópicos, tanto en su potencia como en su forma farmacéutica (loción, crema, pomada, ungüento...). La utilización de uno u otro depende de la zona afectada y del estado de la piel. De igual forma, se encuentran las recomendaciones generales, como lo son: realizar baño diario con agua tibia de máximo 15 minutos de duración. Utilizar geles de ducha y baño sin jabón, denominados syndet, que no irritan la piel. Secar suavemente sin frotar. Hidratar la piel inmediatamente después del baño. Emplear productos emolientes/hidratantes sin perfume e hipo alergénicos, entre otros.

Palabras Claves: Dermatitis Atópica; Eczema Infantil; Dermatitis; Corticoides; Lesiones de Piel. 


\title{
Tratamiento y cuidados de los infantes menores de 2 años con dermatitis atópica
}

Vol. 3, núm. 3 Esp., (2019)

Juan Patricio Méndez Castro; Xiomara Eunisse Mejía Escalante; Olivia Mabell Briones Moreno; Manuel Alejandro Ochoa Medina; Javier Alejandro Sandoval Lema; Bryan Anthony Guerra Jaime

\begin{abstract}
Atopic dermatitis is the most common skin disease in childhood. Approximately $20 \%$ of children under two years suffer from it, an incidence that has increased markedly in developed countries in recent years. This pathology is also recognized, with the name of infantile Eczema; Dermatitis atopic children; Eczema - atopic - children. It is an inflammatory skin disease that manifests with reddish lesions that produce itching. They usually appear in rounded areas in babies (chin, knees, elbows, forehead) and inside the elbows, behind the knees, feet, hands, torso and neck in children. It represents a condition that affects the quality of life of the child so it must be taken immediately to the specialist, so that it immediately indicates dermatological treatment, taking into account that, for this, they must be taken into account, on the one hand, general care and on the other, treatments that include topically applied drugs or that are administered orally. In general, the indicated treatments are topical corticosteroids, which are used to reduce inflammation of the skin, which reduces itching and scratching. There is a wide variety of topical corticosteroids, both in their potency and in their pharmaceutical form (lotion, cream, ointment, ointment ...). The use of one or the other depends on the affected area and the condition of the skin. Similarly, there are general recommendations, such as: perform a daily bath with warm water for a maximum of 15 minutes. Use shower and bath gels without soap, called syndet, that do not irritate the skin. Dry gently without rubbing. Hydrate the skin immediately after bathing. Use emollient / moisturizing products without perfume and hypoallergenic, among others.
\end{abstract}

KeyWords: Atopic dermatitis; Infant Eczema; Dermatitis; Corticosteroids; Skin lesions. 


\section{Tratamiento y cuidados de los infantes menores de 2 años con dermatitis \\ atópica}

Vol. 3, núm. 3 Esp., (2019)

Juan Patricio Méndez Castro; Xiomara Eunisse Mejía Escalante; Olivia Mabell Briones Moreno;

Manuel Alejandro Ochoa Medina; Javier Alejandro Sandoval Lema; Bryan Anthony Guerra

Jaime

\section{Introducción.}

La dermatitis atópica es un trastorno inflamatorio crónico de la piel que se manifiesta en forma de brotes de inflamación (eccema) de forma recurrente. (Cáceres, 2015). De manera general, esta enfermedad se presenta en niños con una predisposición genética y, frecuentemente, aquellos infantes que tienen antecedentes familiares o personales de asma, rino conjuntivitis o alergias alimentarias; patologías éstas que representan alteraciones produciendo una alteración de la barrera cutánea y una inflamación permanente de la epidermis.

Esta enfermedad, es reconocida por los especialistas como un trastorno de la piel a largo plazo, es decir, (crónico) que consiste en erupciones pruriginosas y descamativas. También, se la llama el eczema. En otras palabras, es una condición que se debe a una reacción hipersensible de la piel la cual es similar a una alergia, originando una inflamación permanente de la piel; de manera general, es más común en bebés y en niños, y puede aparecer tan pronto como la edad de 2 a 6 meses, siendo anecdótico de la misma, que muchas personas lo superan en la edad adulta temprana. (Cáceres, 2015)

En términos generales la dermatitis aguda representa una condición que, de manera común es muy difícil de controlar, más aún cuando el paciente es un bebé o un niño, situación ésta que lleva a que la madre sea constante con las indicaciones y tratamiento médico dado por el especialista, contribuyendo, a través de sus cuidados, a evitar que la condición empeore cada vez más y ponga el riesgo de que la piel se inflame generando lesiones en el paciente. Tal y como lo señala (Bernaldt, 2016), al referir que el término atopía representa:

Una condición hereditaria que predispone a padecer una serie de enfermedades, en su mayoría relacionadas con respuestas mediadas por $\operatorname{IgE}$ y alteraciones de la inmunidad celular y de la reactividad vascular. La dermatitis atópica es un conjunto de anomalías y lesiones cutáneas que aparecen en los individuos atópicos. (p.12). 


\section{Tratamiento y cuidados de los infantes menores de 2 años con dermatitis atópica}

Vol. 3, núm. 3 Esp., (2019)

Juan Patricio Méndez Castro; Xiomara Eunisse Mejía Escalante; Olivia Mabell Briones Moreno; Manuel Alejandro Ochoa Medina; Javier Alejandro Sandoval Lema; Bryan Anthony Guerra Jaime

Lo señalado lleva a comprender que, la prevalencia de la dermatitis puede varias; esta condición en muchos pacientes va a depender de los climas y zonas geográficas de donde provenga el mismo. Esta enfermedad se manifiesta, a través de la alteración de la barrera cutánea observada en que su piel, debido a que la misma tiene un tacto especialmente áspero, de aspecto seco y la inflamación crónica produce picor.

De forma periódica presentan fases de empeoramiento, con aparición de áreas de inflamación visible (eccema) caracterizadas por enrojecimiento e incremento del picor. Suelen aparecer de forma simétrica, y las áreas de afectación varían con la edad del niño. (Cerrat, 2014). En lactantes suele ser más frecuente en las mejillas, cuello y zona de extensión de codos y rodillas. En niños mayores de dos años suelen ser más frecuentes en las zonas de flexión de estas regiones y en el tronco. Es un trastorno cuyas manifestaciones suelen mejorar a lo largo de la infancia.

De manera común, los niños con dermatitis atópica tienen una susceptibilidad mayor que otros niños de padecer infecciones de la piel, ya sea por bacterias, virus u hongos, así como de dermatitis de contacto, por la aplicación de algunos fármacos tópicos; esto, debido a que, la dermatitis atópica es una enfermedad que, va en correlación con la gravedad de los síntomas y con el grado de control de la misma, puede alterar la calidad de vida de los niños y de sus familias. (Cerrat, 2014). Esta afectación viene condicionada, además de por el picor, por los problemas con la ropa, con la actividad física, por la alteración del sueño, de la imagen corporal por las lesiones y por la incomodidad de la aplicación de algunos tratamientos; manifestaciones y síntomas éstos que llevan a considerar la importancia del presente artículo, el cual centra su propósito en analizar el tratamiento y cuidados de los infantes menores de 2 años con esta patología.

\section{Fuentes Documentales.}

El análisis correspondiente a las características que predomina en el tema seleccionado, llevan a incluir diferentes fuentes documentales encargadas de darle el respectivo apoyo y en ese sentido cumplir con la valoración de los hechos a fin de generar nuevos criterios que sirven de referencia a otros procesos investigativos. Para (Arias, 2010), las fuentes documentales incorporadas en la 


\section{Tratamiento y cuidados de los infantes menores de 2 años con dermatitis \\ atópica}

Vol. 3, núm. 3 Esp., (2019)

Juan Patricio Méndez Castro; Xiomara Eunisse Mejía Escalante; Olivia Mabell Briones Moreno;

Manuel Alejandro Ochoa Medina; Javier Alejandro Sandoval Lema; Bryan Anthony Guerra

Jaime

investigación documental o bibliográfica, "representa la suma de materiales sistemáticos que son revisados en forma rigurosa y profunda para llegar a un análisis del fenómeno". (p.41). Por lo tanto, se procedió a cumplir con la realización de una lectura previa determinada por encontrar aquellos aspectos estrechamente vinculados con la "TRATAMIENTO Y CUIDADOS DE LOS INFANTES MENORES DE 2 AÑOS CON DERMATOTIS ATÓPICA” para luego explicar mediante un desarrollo las respectivas apreciaciones generales de importancia.

\section{Técnicas para la Recolección de la Información.}

La conducción de la investigación para ser realizada en función a las particularidades que determinan a los estudios documentales, tiene como fin el desarrollo de un conjunto de acciones encargadas de llevar a la selección de técnicas estrechamente vinculadas con las características del estudio. En tal sentido, (Arias Ob cit) refiere, que es "una técnica particular para aportar ayuda a los procedimientos de selección de las ideas primarias y secundarias”. (p. 71).

Por ello, se procedió a la utilización del subrayado, resúmenes, fichaje, como parte básica para la revisión y selección de los documentos que presentan el contenido teórico. Es decir, que mediante su aplicación de estas técnicas se pudo llegar a recoger informaciones en cuanto a la revisión bibliográfica de los diversos elementos encargados de orientar el proceso de investigación. Tal como lo expresa, (Arias Ob cit) "las técnicas documentales proporcionan las herramientas esenciales y determinantes para responder a los objetivos formulados y llegar a resultados efectivos" (p. 58). Es decir, para responder con eficiencia a las necesidades investigativas, se introdujeron como técnica de recolección el método inductivo, que hizo posible llevar a cabo una valoración de los hechos de forma particular para llegar a la explicación desde una visión general.

Asimismo, se emplearon las técnicas de análisis de información para la realización de la investigación que fue ejecutada bajo la dinámica de aplicar diversos elementos encargados de determinar el camino a recorrer por el estudio, según, (Arias, Ob cit) las técnicas de procesamiento de datos en los estudios documentales "son las encargadas de ofrecer al investigador la visión o pasos que debe cumplir durante su ejercicio, cada una de ellas debe estar en correspondencia con 


\section{Tratamiento y cuidados de los infantes menores de 2 años con dermatitis atópica}

Vol. 3, núm. 3 Esp., (2019)

Juan Patricio Méndez Castro; Xiomara Eunisse Mejía Escalante; Olivia Mabell Briones Moreno; Manuel Alejandro Ochoa Medina; Javier Alejandro Sandoval Lema; Bryan Anthony Guerra Jaime

el nivel a emplear" (p. 123). Esto indica, que, para llevar a cabo el procesamiento de los datos obtenidos, es necesario establecer las técnicas que serán seleccionadas, destacándose en este caso, de manera particular: fichas de resumen, textual, registros descriptivos entre otros, los mismos se deben ajustar al nivel que ha sido seleccionado.

\section{Resultados.}

\section{Dermatitis Atópica.}

La dermatitis atópica infantil representa una enfermedad caracterizada por afecciones que se producen en la piel; esta patología suele ser más común en bebés y niños que, en adultos Este periodo de afectación, suele considerarse con un inicio hacia los dos años y un final entre los siete años y la pubertad. (Cerrat, 2014). Las lesiones características se observan sobre todo en las flexuras, en especial en los codos y las rodillas, pero pueden aparecer en otras zonas. En esta fase es más fácil ver lesiones eccematosas con vesículas, pero el intenso prurito hace que enseguida se transformen en erosiones, con exudación y formación de costras.

Las manifestaciones clínicas típicas de la dermatitis atópica se dividen en tres etapas, que suelen denominarse del lactante, infantil y del adulto. Junto a ellas se encuentran otras, con frecuencia llamadas atípicas, a pesar de que muchas, como la xerosis, son muy constantes, tal y como se observa en diversas enfermedades cutáneas y extra cutáneas, las cuales se asocian de forma significativa a la dermatitis atópica. (Darret, 2016) 


\section{Tratamiento y cuidados de los infantes menores de 2 años con dermatitis}

atópica

Vol. 3, núm. 3 Esp., (2019)

Juan Patricio Méndez Castro; Xiomara Eunisse Mejía Escalante; Olivia Mabell Briones Moreno;

Manuel Alejandro Ochoa Medina; Javier Alejandro Sandoval Lema; Bryan Anthony Guerra

Jaime

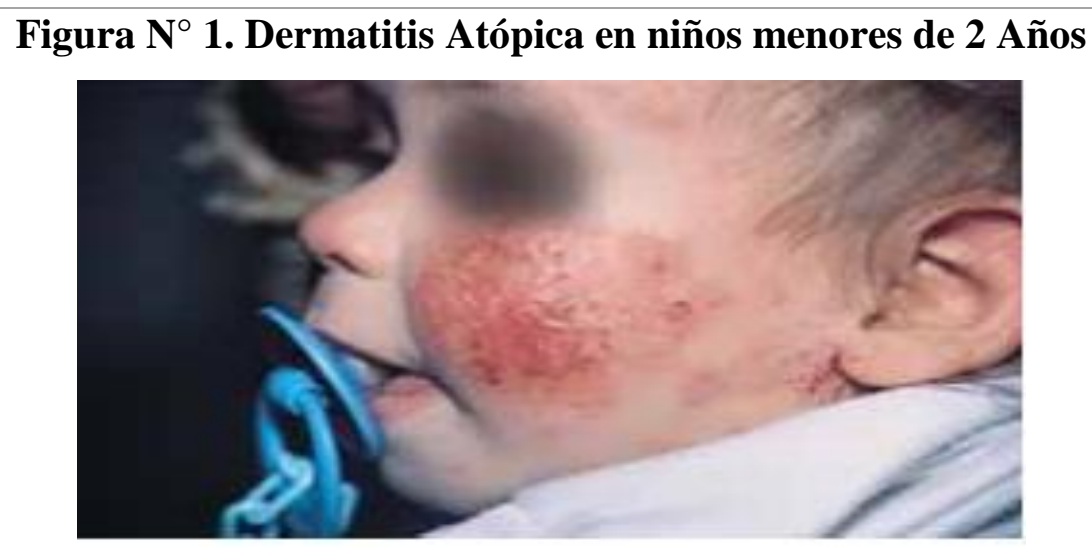

El diagnóstico de la dermatitis atópica se basa en la clínica y su diagnóstico, el cual es manejado, a través de criterios diversos, que son muy empleados, a través de estudios epidemiológicos y ensayos clínicos. (Darret, 2016). La histopatología puede ser útil en casos que planteen problemas de diagnóstico diferencial, este estudio, permite identificar cuando una dermatitis se encuentra en un estado espongiforme (eccema), la mayoría de las veces en un estadio subagudo.

Para el diagnóstico de esta enfermedad no son considerados los datos observados en exámenes de laboratorio; esto, debido a que, los mismos son inconstantes y poco específicos; destacándose, que, en muchos de los casos, estos niños tienen eosinofilia periférica y elevación de la IgE, siendo la determinación de esta última no justificada. (Darret, 2016). De igual forma, tampoco es muy válido el realizar pruebas con antígenos o de IgE específica, tomando en cuenta que, no existan otras manifestaciones clínicas de hipersensibilidad susceptibles de una intervención terapéutica; de ahí, que la morfología y distribución de las lesiones cutáneas de la dermatitis atópica son observadas, a través de las siguientes lesiones elementales: eczema, prúrigo y liquenificación.

- Eczema: caracterizado por zonas de eritema, edema, vesiculación, exudación y costras.

- Prurigo: constituido por pequeñas pápulas con vesícula en su cúspide, que desaparece rápidamente por el rascado, siendo sustituida por una pequeña costra.

- Liquenificación: placas mal delimitadas, engrosadas, recorridas por surcos que delimitan áreas romboidales brillantes. 


\section{Tratamiento y cuidados de los infantes menores de 2 años con dermatitis atópica}

Vol. 3, núm. 3 Esp., (2019)

Juan Patricio Méndez Castro; Xiomara Eunisse Mejía Escalante; Olivia Mabell Briones Moreno; Manuel Alejandro Ochoa Medina; Javier Alejandro Sandoval Lema; Bryan Anthony Guerra Jaime

Lo descrito lleva a considerar que, esta enfermedad se caracteriza a por lesiones de dermatitis, prurito y piel seca (xerosis) que evolucionan con curso crónico y brotes intermitentes alternando con fases de remisión. Asimismo, se logra establecer que las diversas lesiones elementales pueden coexistir en un mismo paciente en un determinado momento de la evolución o sucederse en el tiempo. Todo ello se desarrolla sobre una base de xerosis o piel seca. (Darret, 2016)

\section{Tabla $\mathbf{N}^{\circ} 1$ Criterios diagnósticos de la dermatitis atópica}

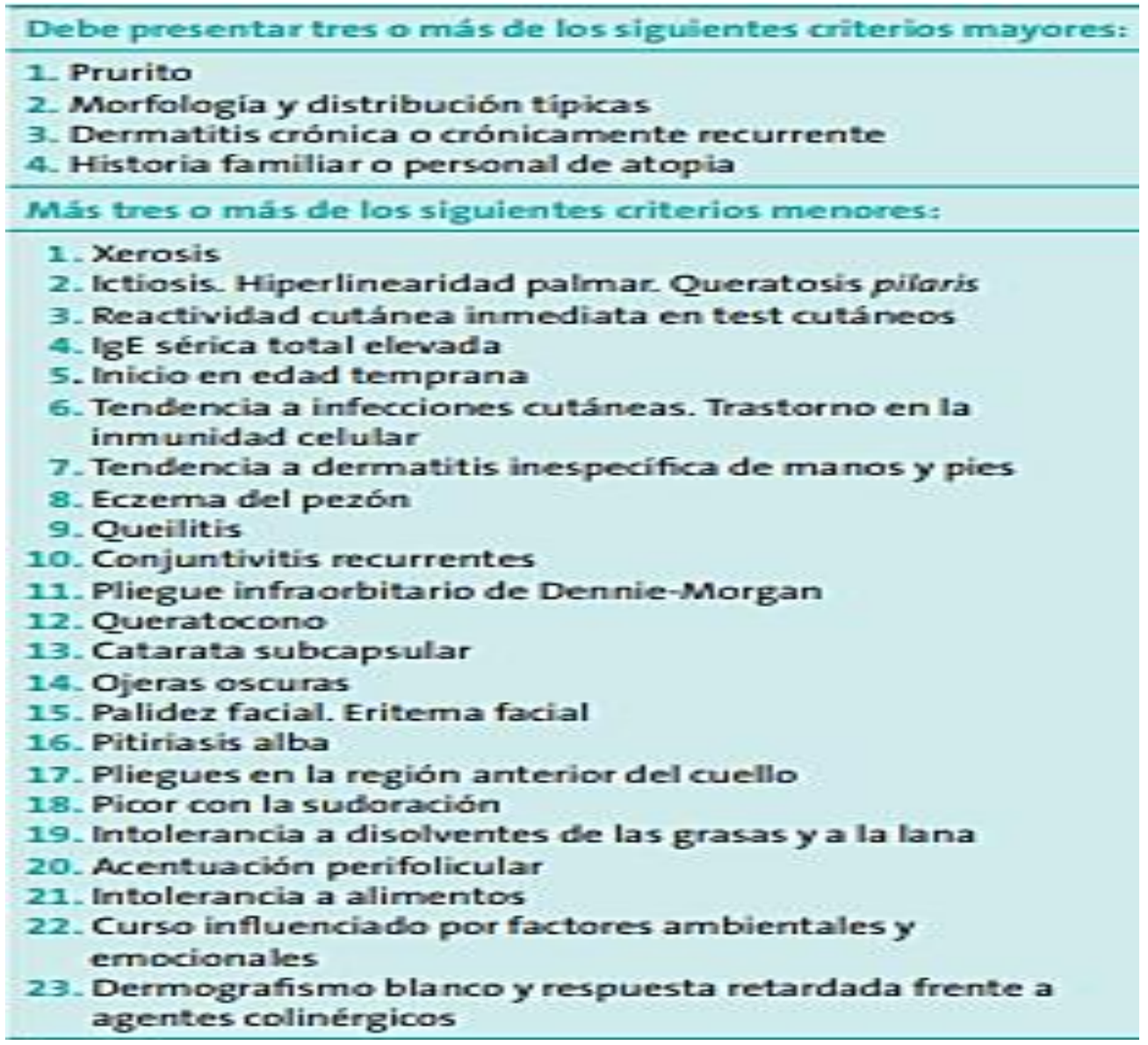

Fuente: (Darret, 2016)

Lo anteriormente lleva a destacar que la dermatitis atópica representa una de las enfermedades cutáneas crónicas más frecuente en la edad pediátrica y afecta aproximadamente a un $10 \%$ de los niños en alguna etapa de su vida. La DA es primariamente una enfermedad propia de la infancia. 


\section{Tratamiento y cuidados de los infantes menores de 2 años con dermatitis}

atópica

Vol. 3, núm. 3 Esp., (2019)

Juan Patricio Méndez Castro; Xiomara Eunisse Mejía Escalante; Olivia Mabell Briones Moreno;

Manuel Alejandro Ochoa Medina; Javier Alejandro Sandoval Lema; Bryan Anthony Guerra

Jaime

Se inicia generalmente a partir de las 6-8 semanas de vida. En el 60\% de los pacientes se inicia en el primer año y solo el 10\% inicia la enfermedad después de cumplir los siete años. (Darret, 2016). Desde el punto de vista fisiopatológico, se caracteriza por un deterioro de la función barrera cutánea y por la inflamación debida a la interacción entre un antígeno actualmente desconocido, las células de presentación de antígenos y las células T.

La fase de lactante se caracteriza por lesiones eccematosas muy pruriginosas con gran componente exudativo que forma costras. Inicialmente, se localiza en las mejillas (Imagen $\mathrm{N}^{\circ} 2$ ) desde donde puede extenderse a la frente, los pliegues auriculares y el cuero cabelludo, pero respetando el triángulo nasolabial. (Darret, 2016). Desde la región facial, con el tiempo puede extenderse al resto del cuerpo, afectando de forma característica a la superficie extensora de los miembros.

\section{Imagen $\mathbf{N}^{\circ}$ 2. Dermatitis Atópica. Fase de Lactante}

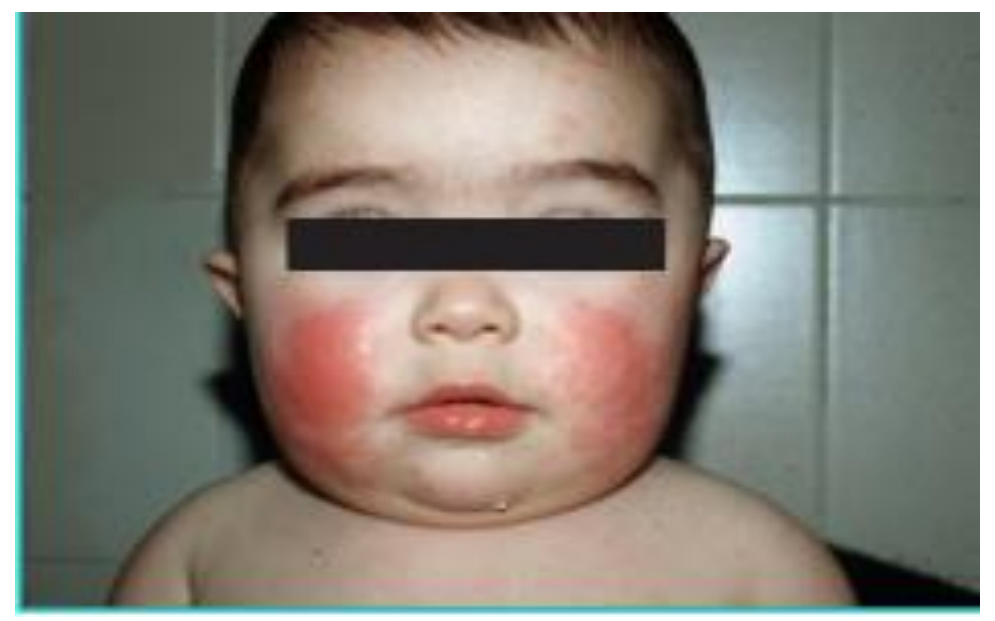

Fuente: (Darret, 2016)

En la fase infantil, que se extiende desde los dos años hasta la pubertad, las lesiones se hacen más papulosas y menos exudativas, con tendencia a la liquenificación activada por el rascado, y tienden a localizarse de forma característica en los pliegues, sobre todo antecubital y poplíteo (Figura 3). También pueden afectar a la región palpebral y a la peribucal, a los pliegues auriculares, a las muñecas, a las manos, al pulpejo de los dedos, a los tobillos y a los pies (afectando sobre todo al 


\section{Tratamiento y cuidados de los infantes menores de 2 años con dermatitis atópica}

Vol. 3, núm. 3 Esp., (2019)

Juan Patricio Méndez Castro; Xiomara Eunisse Mejía Escalante; Olivia Mabell Briones Moreno; Manuel Alejandro Ochoa Medina; Javier Alejandro Sandoval Lema; Bryan Anthony Guerra Jaime

dorso de los dedos y al tercio anterior de la región plantar del pie, con afectación característica del primer dedo).

\section{Tratamiento.}

El tratamiento incluye varias medidas, sobre todo centradas en los cuidados generales de la piel y el tratamiento farmacológico, tanto de los brotes como, en caso necesario, crónico. Antes de iniciar el tratamiento es esencial explicar con detalle a la familia del niño qué son la atopia y la dermatitis atópica, qué pronóstico tienen y qué puede esperarse del tratamiento. (Death, 2016). Es muy importante aclarar que la dermatitis atópica no es una alergia a ninguna sustancia determinada y que en ningún modo va a resolverse por realizar "pruebas alérgicas" ni por eliminar alimentos. Asimismo, deben evitarse todas las circunstancias y elementos que provocan prurito, como el calor, especialmente el producido por aire caliente, la sequedad ambiental, el contacto con lana, plásticos y en algunos casos otras fibras.

Si bien el exceso de agua y jabón es perjudicial, también lo es la falta de higiene. Es preferible realizar duchas que baños, cortas, con agua no excesivamente caliente y con un gel de pH ácido. A continuación, se aplicará una crema emoliente en todo el cuerpo, repitiéndola en otros momentos del día en las zonas de xerosis. (Death, 2016). Las medidas para evitar irritantes incluyen cuidados para disminuir el contacto con la piel de alimentos ácidos (tomate, cítricos, etc.), cambio frecuente de pañales y aclarado cuidadoso de la ropa. Algunos alimentos ricos en histamina, en cantidades considerables y en niños muy sensibles, pueden agravar el prurito (fresas, frutos secos, mariscos, etc.). Igual efecto puede tener algunos medicamentos liberadores de histamina (ácido acetil salicílico, codeína) o vasodilatadores.

Terapéutica tópica En el momento actual la base del tratamiento tópico son los corticoides, que bien empleados consiguen un control satisfactorio de la inmensa mayoría de los casos. La elección del corticoide y el excipiente debe adecuarse a la lesión y la zona a tratar. Para las lesiones habituales en los niños, los corticoides de potencia baja o media suelen ser suficiente. El clobetasol no debe utilizarse en la infancia. (Death, 2016). En la cara, los pliegues, las mamas y la región 


\section{Tratamiento y cuidados de los infantes menores de 2 años con dermatitis \\ atópica}

Vol. 3, núm. 3 Esp., (2019)

Juan Patricio Méndez Castro; Xiomara Eunisse Mejía Escalante; Olivia Mabell Briones Moreno;

Manuel Alejandro Ochoa Medina; Javier Alejandro Sandoval Lema; Bryan Anthony Guerra

Jaime

genital deben usarse preparados de perfil de seguridad alto, para evitar efectos indeseados, como rosácea, atrofia, estrías, telangiectasias, hipertricosis, etc. En niños mayores de 2 años los inhibidores tópicos de la calcineurina suponen una alternativa a la terapéutica con esteroides. El pimecrolimus puede utilizarse en las fases prodrómicas e iniciales para evitar la progresión del brote. El tacrolimus puede utilizarse en casos de intensidad moderada o grave, rebeldes al tratamiento convencional o en los que los corticoides tópicos estén contraindicados.

Terapéutica sistémica Los antihistamínicos orales, especialmente los de primera generación que poseen un efecto sedante, son una medida coadyuvante de los corticoides tópicos de gran utilidad. Los antibióticos activos frente a Staphylococcus aureus pueden ayudar a controlar los brotes agudos, especialmente si hay sobreinfección evidente. Los corticoides orales sólo deben usarse en casos rebeldes a los tratamientos anteriores y du rante periodos cortos. (Death, 2016). Si son necesarios, es preferible el empleo de prednisona o metilprednisolona que dexametasona. Sólo en casos excepcionales es preciso recurrir a otras medidas, como la administración de otros inmunosupresores o inmuno moduladores sistémicos (ciclosporina, metotrexato, azatioprina, tacrolimus, etc) o a antidepresivos tricíclicos, para controlar el prurito. En niños mayores de 13 años puede valorarse la foto quimioterapia (PUVA)

\section{Cuidados Generales de la Piel.}

Cuando se habla de cuidados de la piel se está haciendo referencia al control de los factores ambientales que modifican la expresión de la enfermedad (tales como la temperatura, el grado de humedad y de contaminación ambiental) y a la higiene e hidratación diaria de la piel. En cuanto a los factores ambientales, el frío y la sequedad ambiental deshidratan la piel del niño atópico, de ahí que la mayoría suelan empeorar en invierno y mejorar en verano. En base a esto, se señalan las siguientes recomendaciones:

- El sol y la humedad ambiental disminuyen la inflamación de la piel atópica, así como también los baños en agua del mar. 


\section{Tratamiento y cuidados de los infantes menores de 2 años con dermatitis atópica}

Vol. 3, núm. 3 Esp., (2019)

Juan Patricio Méndez Castro; Xiomara Eunisse Mejía Escalante; Olivia Mabell Briones Moreno; Manuel Alejandro Ochoa Medina; Javier Alejandro Sandoval Lema; Bryan Anthony Guerra Jaime

- Es recomendable que el baño o ducha sean a diario, incluso en épocas en que tengan lesiones de eccema activas, pues contribuye a disminuir el picor, a relajar al niño y a limpiar y descontaminar la piel.

- Se recomienda utilizar jabones de pH ácido, dado que el pH normal de la piel es de 3,55,5 , secar la piel sin frotarla y, cuando aún esté húmeda, aplicar la crema hidratante o los fármacos tópicos.

- Se recomienda que la ropa sea amplia y de algodón o lino, que permitan la transpiración. Ha de lavarse con detergentes suaves y aclararse bien para que no queden restos que puedan irritar la piel.

- Asimismo, es importante retirar las etiquetas o superficies rugosas que puedan estar en contacto con la piel e irritarla por fricción o por mecanismo químico.

- La elección del producto utilizado para la hidratación de la piel ha de basarse en la facilidad de aplicación, en su coste y en las preferencias personales o familiares, pues la constancia en la aplicación diaria, cuyo cumplimiento depende de estos factores, reduce los brotes y mejora la calidad de vida de los pacientes. (Death, 2016)

Por otra parte, se encuentran los cuidados relacionados con las normas de alimentación, destacándose entre las mismas: que, la alimentación del niño con dermatitis atópica debe ser la normal para su edad y únicamente está indicada una dieta de exclusión cuando se haya demostrado una alergia (por las manifestaciones del niño y por pruebas de alergia) a algún alimento concreto.

Seguido a esto, se establece lo relacionado al tratamiento farmacológico el cual se desarrollará de acuerdo a lo referido por el médico tratante en sus prescripciones médicas, los mismos entre sus propósitos buscan disminuir la inflamación de la piel durante los brotes, mejorar los síntomas (picor, alteración del sueño y de la calidad de vida) y disminuir el número de brotes o recurrencias.

- Los corticoides tópicos son los fármacos más utilizados en el tratamiento de los brotes. Se utilizan para disminución de la inflamación de la piel, por lo que disminuyen el picor y el rascado. Existe una gran variedad de corticoides tópicos, tanto en su potencia como en su 


\section{Tratamiento y cuidados de los infantes menores de 2 años con dermatitis}

atópica

Vol. 3, núm. 3 Esp., (2019)

Juan Patricio Méndez Castro; Xiomara Eunisse Mejía Escalante; Olivia Mabell Briones Moreno;

Manuel Alejandro Ochoa Medina; Javier Alejandro Sandoval Lema; Bryan Anthony Guerra

Jaime

forma farmacéutica (loción, crema, pomada, ungüento...). La utilización de uno u otro depende de la zona afectada y del estado de la piel.

- Los inhibidores de la calcineurina tópicos (tacrolimus y pimecrolimus) son fármacos inmunomoduladores que han demostrado eficacia y seguridad tanto para el tratamiento de los brotes como para prevenir su aparición, como tratamiento de mantenimiento preventivo.

- Los antihistamínicos orales tienen como finalidad disminuir el picor. Son de elección los antihistamínicos de primera generación (hidroxicina, dexclorfeniramina), por su efecto sedante. No se recomienda el uso de antihistamínicos tópicos dado que pueden producir fotosensibilidad.

- En casos de sobreinfección de la piel puede ser necesario el tratamiento con asociaciones de corticoides y antibióticos o antifúngicos tópicos.

- Los corticoides y los inmunomoduladores sistémicos son medicamentos de uso excepcional, en casos graves o que no mejoran con el tratamiento tópico. (Death, 2016)

\section{Cuidados para la picazón de la Piel.}

La picazón severa es común. Esta puede comenzar incluso antes de que aparezca la erupción. La dermatitis atópica se llama a menudo "erupciones que pican" debido a que primero comienza la picazón, y luego sigue la erupción de la piel como consecuencia del rascado. Para ayudar a su hijo a evitar rascarse:

- Use una crema hidratante, crema con esteroides tópicos, u otros medicamentos que prescriba el proveedor del niño.

- Mantenga las uñas del niño cortas. Haga que use guantes livianos mientras duerme si rascarse por la noche es un problema.

- Déle antihistamínicos u otros medicamentos por vía oral, según lo indicado por el proveedor de su hijo.

- Tanto como sea posible, enseñar a los niños de más edad a no rascarse la piel irritada. (Death, 2016) 


\section{Tratamiento y cuidados de los infantes menores de 2 años con dermatitis atópica}

Vol. 3, núm. 3 Esp., (2019)

Juan Patricio Méndez Castro; Xiomara Eunisse Mejía Escalante; Olivia Mabell Briones Moreno; Manuel Alejandro Ochoa Medina; Javier Alejandro Sandoval Lema; Bryan Anthony Guerra Jaime

\section{Cuidado de la piel diario.}

El cuidado de la piel todos los días con productos libres de alérgenos puede reducir la necesidad de medicamentos, para el logro de ello se recomienda:

- Utilice pomadas hidratantes (como vaselina), cremas o lociones.

- Elija productos para la piel que se fabrican para personas con eczema o piel sensible. Estos productos no contienen alcohol, aromas, colorantes y otros productos químicos.

- Tener un humidificador para mantener la humedad del aire también ayudará.

- Las cremas hidratantes y emolientes funcionan mejor cuando se aplican a la piel que está mojada o húmeda.

- Después de lavarse o bañarse, acaricie la piel seca y luego aplique la crema hidratante de inmediato.

- $\mathrm{Su}$ proveedor también puede recomendar la colocación de un vendaje sobre estos ungüentos hidratantes para la piel. (Death, 2016)

\section{Al lavar o bañar a su hijo:}

- Báñelo con menos frecuencia y que el contacto con el agua sea lo más más breve posible. Los baños cortos y más fríos son mejores que los baños calientes prolongados.

- Utilice limpiadores suaves para la piel en lugar de jabones tradicionales, y úselos sólo en la cara, las axilas, las zonas genitales, manos y pies de su niño.

- $\quad$ NO frote ni reseque la piel demasiado fuerte o por demasiado tiempo.

- Justo después del baño, aplique crema, loción o ungüento lubricante mientras la piel está todavía húmeda para mantener la humedad. (Death, 2016)

\section{Recomendaciones Generales:}

- Vista al niño con ropa suave y cómoda, como ropa de algodón.

- Que el niño beba mucha agua. Esto puede ayudar a aumentar la humedad a la piel.

- Enseñe a los niños de más edad estos mismos consejos para el cuidado de la piel. 


\section{Tratamiento y cuidados de los infantes menores de 2 años con dermatitis \\ atópica}

Vol. 3, núm. 3 Esp., (2019)

Juan Patricio Méndez Castro; Xiomara Eunisse Mejía Escalante; Olivia Mabell Briones Moreno;

Manuel Alejandro Ochoa Medina; Javier Alejandro Sandoval Lema; Bryan Anthony Guerra

Jaime

- La erupción en sí, así como el rascado, a menudo causan heridas en la piel y pueden conducir a infección.

- Observe por señales de enrojecimiento, calor, hinchazón, u otros signos de infección.

- Llame al proveedor de su hijo a la primera señal de infección. (Death, 2016)

Evite disparadores.

Los siguientes factores desencadenantes pueden hacer que los síntomas de la dermatitis atópica empeores:

- Alergias al polen, moho, ácaros del polvo, o de los animales

- El aire frío y seco en el invierno

- Resfriados o la gripe

- El contacto con sustancias irritantes y sustancias químicas

- El contacto con materiales muy gruesos, tales como lana

- Piel seca

- Estrés emocional

- Los baños frecuentes o ducha y la natación a menudo, lo que puede secar la piel

- Estar demasiado caliente o demasiado frío, así como cambios bruscos de temperatura

- Perfumes o colorantes añadidos a lociones o jabones para la piel (Death, 2016)

- Para prevenir los brotes, trate de evitar:

- Alimentos como huevos, que pueden causar una reacción alérgica en un niño muy pequeño. Siempre hable con su proveedor primero.

- Lana, lanolina y otras telas que pican. Utilice, ropa de textura lisa y ropa de cama, como de algodón.

- Transpiración: Tenga cuidado de no vestir de más a su hijo durante épicas cálidas.

- Los jabones fuertes o detergentes, así como productos químicos y disolventes.

- Los cambios repentinos en la temperatura corporal, que pueden causar sudoración y empeorar la condición de su hijo. 


\section{Tratamiento y cuidados de los infantes menores de 2 años con dermatitis atópica}

Vol. 3, núm. 3 Esp., (2019)

Juan Patricio Méndez Castro; Xiomara Eunisse Mejía Escalante; Olivia Mabell Briones Moreno; Manuel Alejandro Ochoa Medina; Javier Alejandro Sandoval Lema; Bryan Anthony Guerra Jaime

- Estrés: Observe si hay señales de que su hijo se siente frustrado o estresado y enséñele maneras de reducir el estrés, tales como tomar respiraciones profundas o pensar en las cosas que disfrutan.

- Disparadores que causan los síntomas de la alergia: Haga lo que pueda para mantener su hogar libre de los desencadenantes de alergias como el moho, el polvo y la caspa de mascotas.

- $\quad$ Evite el uso de productos de cuidado de la piel que contienen alcohol. (Death, 2016)

El uso de cremas hidratantes, cremas, o ungüentos todos los días según las indicaciones puede ayudar a prevenir las erupciones.

\section{Medicamentos que indica el médico.}

Los antihistamínicos tomados por vía oral pueden ayudar si las alergias causan picazón en la piel de su hijo. Estos medicamentos a menudo son de venta libre y no requieren receta médica. Consulte con el proveedor de su hijo qué tipo es adecuado para su hijo, considerándose que, la dermatitis atópica suele tratarse con medicamentos aplicados directamente sobre la piel o el cuero cabelludo. Estos son los llamados medicamentos tópicos:

- El proveedor probablemente le recetará una crema o pomada de cortisona (esteroide) leve al principio. Los esteroides tópicos contienen una hormona que ayuda a "calmar" la piel del niño cuando está hinchada o inflamada. Su niño puede necesitar un medicamento más fuerte si esto no funciona.

- Los medicamentos llamados inmuno moduladores tópicos se pueden prescribir para cualquier persona mayor de 2 años.

- Las cremas o ungüentos que contengan alquitrán de hulla o antralina se pueden utilizar para las áreas engrosadas de la piel.

- Las cremas hidratantes y cremas que restauran la barrera de la piel también son útiles. (Death, 2016) 


\section{Tratamiento y cuidados de los infantes menores de 2 años con dermatitis}

atópica

Vol. 3, núm. 3 Esp., (2019)

Juan Patricio Méndez Castro; Xiomara Eunisse Mejía Escalante; Olivia Mabell Briones Moreno;

Manuel Alejandro Ochoa Medina; Javier Alejandro Sandoval Lema; Bryan Anthony Guerra

Jaime

Otros tratamientos que se pueden usar incluyen:

- Cremas o pastillas antibióticas si la piel de su niño está infectada

- Medicamentos que suprimen el sistema inmunológico

- Fototerapia, un tratamiento médico en el que la piel del niño se expone cuidadosamente a la luz ultravioleta (UV)

- El uso a corto plazo de esteroides sistémicos (esteroides administrados por vía oral oral o a través de una vena). (Death, 2016)

\section{Cuándo llamar al médico Tratante:}

Llame al Médico tratante de su hijo si:

- La dermatitis atópica no mejora con el cuidado en el hogar

- Los síntomas empeoran o el tratamiento no funciona

- Su niño tiene signos de infección, tales como enrojecimiento, pus o protuberancias llenas de líquido en la piel, fiebre, o dolor. (Death, 2016)

\section{Conclusiones.}

La dermatitis atópica representa una de las enfermedades cutáneas crónicas más frecuente en la edad pediátrica y afecta aproximadamente a un $10 \%$ de los niños en alguna etapa de su vida. La DA es primariamente una enfermedad propia de la infancia. Esta patología de manera común, inicia a partir de las 6-8 semanas de vida. En el 60\% de los pacientes se inicia en el primer año y solo el $10 \%$ inicia la enfermedad después de cumplir los siete años. Desde el punto de vista fisiopatológico, se caracteriza por un deterioro de la función barrera cutánea y por la inflamación debida a la interacción entre un antígeno actualmente desconocido, las células de presentación de antígenos y las células T. 


\section{Tratamiento y cuidados de los infantes menores de 2 años con dermatitis atópica}

Vol. 3, núm. 3 Esp., (2019)

Juan Patricio Méndez Castro; Xiomara Eunisse Mejía Escalante; Olivia Mabell Briones Moreno; Manuel Alejandro Ochoa Medina; Javier Alejandro Sandoval Lema; Bryan Anthony Guerra Jaime

Entre sus tratamientos se encuentra la terapéutica tópica, siendo la base fundamental del tratamiento tópico los corticoides, que bien empleados consiguen un control satisfactorio de la inmensa mayoría de los casos. Para las lesiones habituales en los niños, los corticoides de potencia baja o media suelen ser suficiente. El pimecrolimus puede utilizarse en las fases prodrómicas e iniciales para evitar la progresión del brote. El tacrolimus puede utilizarse en casos de intensidad moderada o grave, rebeldes al tratamiento convencional o en los que los corticoides tópicos estén contraindicados. Por otra parte, se encuentra la terapéutica sistémica, en la cual son utilizados los antihistamínicos orales, especialmente los de primera generación que poseen un efecto sedante, son una medida coadyuvante de los corticoides tópicos de gran utilidad.

Entre los cuidados más recomendados se encuentra el desarrollar la higiene de la piel atópica en niños. En caso de bebés muy pequeños o dermatitis severa puede ser mejor no bañar cada día, sino hacerlo en días alternos, salvo que haya mucha suciedad por sudor, restos de comida, o de arena del parque. Para el baño, el agua no debe estar muy caliente, mejor tibia. Los cambios bruscos de temperatura también pueden resultar irritantes para la piel atópica. Asimismo, se deben utilizar elementos de higiene adecuados, ya que la mayoría de jabones convencionales arrastran la capa protectora de la piel, facilitando la sequedad y el picor. Estos productos se conocen como syndet (sin jabones). Los productos para la higiene diaria deben tener capacidad hidratante y protectora de la piel, de forma que no resulten abrasivos. Tras salir del baño la piel debe secarse con cuidado, dando pequeños toques, evitando el frotado con la toalla. Después del secado iniciaremos la rutina de la hidratación

Para la hidratación de la piel atópica en niños, es recomendable la utilización de cremas hidratantes y emolientes, especialmente aquellas que son formuladas y que no contienen sustancias irritantes, evitan la sequedad de la piel. El momento ideal para hidratar la piel es justo después del baño, aunque en algunos casos puede ser necesario hidratar de forma añadida en algún otro momento del día. Una vez finalizado el baño y con la piel todavía un poco húmeda se aplican los productos hidratantes. Elige productos con capacidad hidratante y emoliente que ayuden a restaurar la barrera natural de la piel, alivien la sensación de prurito y favorezcan el crecimiento de la micro flora 


\section{Tratamiento y cuidados de los infantes menores de 2 años con dermatitis \\ atópica}

Vol. 3, núm. 3 Esp., (2019)

Juan Patricio Méndez Castro; Xiomara Eunisse Mejía Escalante; Olivia Mabell Briones Moreno;

Manuel Alejandro Ochoa Medina; Javier Alejandro Sandoval Lema; Bryan Anthony Guerra

Jaime

habitual de la piel protegiendo de esa forma, las sobreinfecciones. Mantener una rutina de hidratación durante todo el año ayuda a cuidar la piel y a incorporar ese hábito a los cuidados habituales de higiene del niño, de forma que conforme vaya adquiriendo autonomía, pueda ser él mismo quien se aplique el producto.

En cuanto a la ropa y la alimentación del niño que padece de dermatitis atópica se recomienda que, la vestimenta sea de algodón, ya que la lana y los tejidos sintéticos empeoran la piel. De igual forma, deben elegirse jabones hipo alergénicos y sin fragancias. El lavado del vestido debe ser bien enjuagada para retirar restos de jabón y suavizante. En lo referido a la alimentación se postula que, aunque puede existir asociación entre algunas alergias alimentarias y la dermatitis atópica, no deben hacerse dietas de exclusión salvo que haya un diagnóstico de confirmación de la alergia.

\section{Bibliografía.}

Arias, F. (2010). Paradigmas de la Investigación Cientifica. España: Editorial: Luces.

Bernaldt, e. (2016). El Niño con Dermatitis Atópica. Una visión global. Madrid, Loki and Dimas, $17-21$.

Cáceres, L. (2015). Dermatitis Aguda, Tratamientos y Cuidados. Dermatología Pediátrica. Madrid, Aula Médica., 83-90.

Cerrat, P. (2014). Características Clínicas de la Dermatitis Atópica. www.especialistasdermatologia.com., 22-23.

Darret, G. (2016). Dermatitis. Aspectos Clínicos y Tratamiento. Madrid: Editorial: Mosby .

Dávila, N. (2012). Paradigmas de la Investigación Científica. Pereire, Colombia: Editorial: Las Brisas.

Death, C. (2016). Atopi Dermatitis. Dermatology. 4th ed. Philadelphia, PA: Elsevier, 12 - 14. 
Tratamiento y cuidados de los infantes menores de 2 años con dermatitis atópica

Vol. 3, núm. 3 Esp., (2019)

Juan Patricio Méndez Castro; Xiomara Eunisse Mejía Escalante; Olivia Mabell Briones Moreno; Manuel Alejandro Ochoa Medina; Javier Alejandro Sandoval Lema; Bryan Anthony Guerra Jaime

$$
\text { (9) (1)(0) }
$$

\section{RECONOCIMIENTO-NOCOMERCIAL-COMPARTIRIGUAL}

CC BY-NC-SA

ESTA LICENCIA PERMITE A OTROS ENTREMEZCLAR, AJUSTAR Y CONSTRUIR A PARTIR DE SU OBRA CON FINES NO

COMERCIALES, SIEMPRE Y CUANDO LE RECONOZCAN LA AUTORÍA Y SUS NUEVAS CREACIONES ESTÉN BAJO UNA LICENCIA CON LOS MISMOS TÉRMINOS. 